\title{
Toxic Keratoconjunctivitis from Coral Reef
}

\author{
Tedi Begaj $^{a} \quad$ Stephan Ong Tone ${ }^{b} \quad J o s e p h$ B. Ciolino ${ }^{a}$ \\ aDepartment of Ophthalmology, Massachusetts Eye and Ear, Harvard Medical School, \\ Boston, MA, USA; ${ }^{b}$ Cornea and External Eye Disease, Sunnybrook Health Sciences Centre \\ and Sunnybrook Research Institute, Department of Ophthalmology and Vision Sciences, \\ University of Toronto, Toronto, ON, Canada
}

\section{Keywords}

Coral $\cdot$ Zoanthid $\cdot$ Aquarium $\cdot$ Palytoxin $\cdot$ Toxic keratoconjunctivitis

\begin{abstract}
A 25-year-old woman presented with right eye pain, lid edema, conjunctival injection and chemosis, and mild corneal epitheliopathy after exposure to fluid content from an aquarium coral reef. Topical moxifloxacin and prednisolone were started 4 times daily, with full clinical resolution after 2 weeks. Toxin-mediated keratoconjunctivitis may occur after exposure to zoanthid coral reef, particularly in aquarium enthusiasts. Topical corticosteroids in tandem with topical antibiotics appear to be effective in mild disease. However, in severe cases that exhibit corneal infiltrates and stromal thinning, close observation is warranted in case of possible keratolysis.
\end{abstract}

\section{Introduction}

Palytoxin (PTX) is produced by various marine animals, including zoanthids, which are commonly found in coral reefs; it causes cellular toxicity by inducing potent vasoconstriction and blocking the $\mathrm{Na}^{+} / \mathrm{K}^{+}$ATPase [1]. Although rare, human exposure to PTX can cause significant morbidity and mortality, with common symptoms including fever, weakness and neuromuscular dysfunction, respiratory distress, myalgias, various rashes, and gastrointestinal distress [2]. Inhalation exposure from aerosolized PTX can cause bronchial inflammation, spams and may even lead to respiratory failure [3,4]. Cutaneous exposure can result in contact dermatitis-like reaction while consumption of fish that bioaccumulate PTX can lead to various gastrointestinal symptoms (e.g., nausea, vomiting, and diarrhea) as well electrolyte and cardiac conduction abnormalities which could cause death [5].

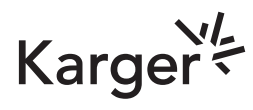


On the ocular surface, PTX causes corneal epithelial disruption and nerve damage [6]. The first case of toxic keratoconjunctivitis - described in 1993 - was due to ocular contact with secretions from a soft coral called Alcyonium digitatum (commonly referred as dead man's finger) [7]. In this small series, patients presented with moderate conjunctival chemosis and hemorrhage as well as a punctate epithelial keratopathy. All patients were irrigated, treated with topical corticosteroids and antibiotics, and ultimately had full recovery. The work herein describes a case of PTX keratoconjunctivitis and provides further evidence that topical corticosteroids in tandem with topical antibiotics may serve as an effective treatment.

\section{Case Presentation}

A healthy 25-year-old woman, with no history of contact lens wear, presented with several hours of pain, injection and tearing in the right eye. The patient reported accidental exposure to coral reef fluid (Zoanthids-Green Bay Packers, Fig. 1) when cleaning her aquarium. She immediately irrigated the eye and continued using artificial tears every 3-4 h.

On examination, before instillation of topical anesthetic, testing of the $\mathrm{pH}$ with a Hydrion (Micro Essential Laboratory Inc., Brooklyn, NY, USA) pH strip in both upper and lower fornices revealed a normal $\mathrm{pH}$ of 7.0. The $\mathrm{pH}$ strip was applied into each fornix for $1 \mathrm{~s}$, then instantly compared the resulting color with the matching pH color chart. Visual acuity was 20/20 in both eyes with normal intraocular pressures. She had upper greater-than-lower lid edema with minimal erythema and no induration. In addition, there was a significant inferior bulbar conjunctival epithelial defect with associated injection, subtle blanching of conjunctival vessels, chemosis, and patchy subconjunctival hemorrhage (Fig. 2a); there was no corneal epithelial defect (Fig. 2b). Conjunctival swabs were sent for bacterial culture and gram stain. She was started on topical moxifloxacin $0.5 \%$ and prednisolone acetate $1.0 \% 4 \times$ daily as well as preservative-free artificial tears every $2 \mathrm{~h}$. The lid edema worsened over the next $24 \mathrm{~h}$, but she remained afebrile with no changes on ocular exam indicating a low likelihood of a secondary bacterial cellulitis.

On follow up 3 days later, her symptoms had significantly improved; similarly, her exam revealed resolution of the lid edema as well as resolution of the conjunctival injection and chemosis. Gram stain and culture of the conjunctiva showed no neutrophils or organisms. At 2-week follow-up, her symptoms had resolved. Moxifloxacin was stopped and she was subsequently tapered off the prednisolone over a 4-week period by reducing 1 drop each week.

\section{Discussion}

Since the first case report published in 1993 [7], there have been additional articles [8-16] (19 cases) published in English (with another 2 published in German [17] and Dutch [18]) discussing the manifestations of ocular exposure to PTX from coral reef. All known and reported cases of PTX keratoconjunctivitis are succinctly described in a table [14]. The majority of publications have come after 2010, highlighting the increased awareness and diagnosis of PTX keratoconjunctivitis. There has also been a report [8] of a young woman with unilateral corneal infiltrates and conjunctival injection after scuba diving next to red coral reef, with complete resolution after topical corticosteroids and antibiotics.

The clinical presentation of PTX keratoconjunctivitis may be confused with an acanthamoeba infection due to stromal ring infiltrates [9]. After exposure to zoanthid coral from a personal aquarium, a patient immediately developed ocular irritation. Treatment with the usual regimen of topical corticosteroids and antibiotics was initiated, but the stromal ring

\section{Karger'}


Fig. 1. Photograph of zoanthid (Green Bay Packers) coral from the patient's aquarium. Individual purple polyps are seen growing together in a colony.
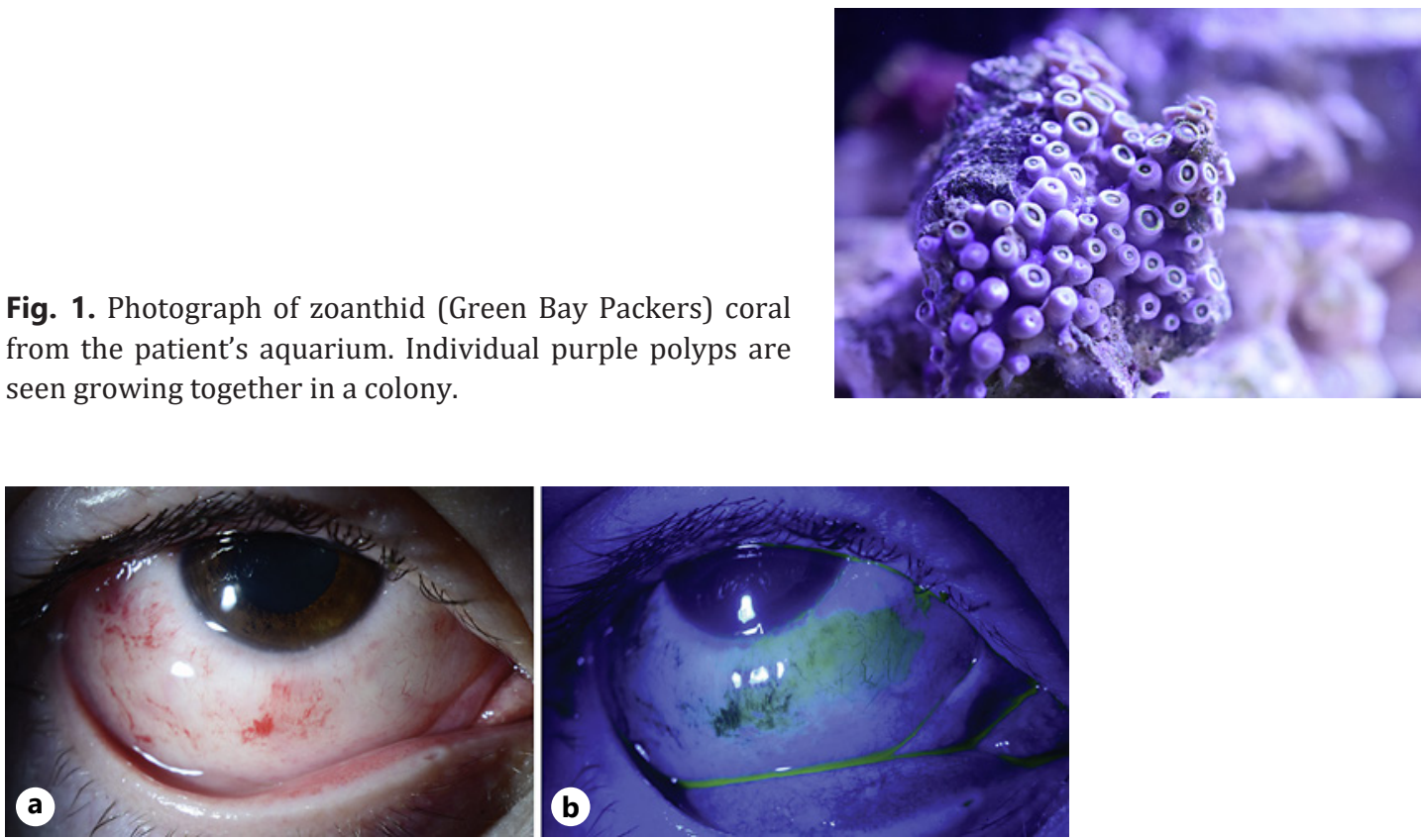

Fig. 2. External photo of the right eye. a Superior lid edema with inferior conjunctival injection, chemosis, and patchy subconjunctival hemorrhage. b Fluorescein staining of the inferior bulbar conjunctiva but no corneal epithelial defect.

infiltrate (and associated epithelial defect) persisted. When the prednisolone drops were increased to hourly dosing, clinical improvement was noted with resolution several weeks later. However, mid-peripheral stromal thinning remained.

The extent of ocular toxicity resulting from PTX-induced toxic keratoconjunctivitis depends on the level and duration of exposure. Although most cases reported in the literature resolve with varying degrees of corneal scarring, a larger series [12] showed that $\sim 57 \%$ of their patients developed progressive corneal melting, with some patients requiring ultimately therapeutic penetrating keratoplasty. In addition to topical corticosteroids, oral doxycycline, prednisone and a tarsorrhaphy were utilized with the goal of arresting progressive corneal thinning.

A robust review of the literature as well as further insight on a severe case of toxic keratoconjunctivitis that led to corneal perforation was published recently [14]. The patient was exposed to a plume of dust after handling coral and initially suffered from both eye pain as well as multi-system involvement of respiratory distress, abdominal pain, and diffuse pruritus. He was monitored in the ICU, and while his systemic symptoms improved, he suffered from progressive peripheral corneal thinning that ultimately required a penetrating keratoplasty.

In general, the diagnosis of PTX-induced keratoconjunctivitis is established by both a clear antecedent exposure to coral content and common clinical characteristics mentioned above. Although there is an increase in awareness of this rare toxicity (particularly in the last decade), there are no current clinical guidelines for treatment after ocular exposure to PTX. The commonality between the published reports consisted of topical steroids and antibiotics. In cases of a robust immune response causing stromal infiltrates, more frequent topical steroids may be warranted. Clinicians must closely monitor their patients as corneal melt with subsequent perforation may occur, necessitating possible surgical intervention.

Our patient presented within hours of ocular exposure to zoanthid coral content with initiation of treatment early in the disease course. Over the next 3 days, she clinically and 
symptomatically improved, requiring no modification of the treatment regimen and no need for escalation to more aggressive therapy. Ultimately, she had full resolution without any corneal thinning or scarring.

\section{Conclusion}

It is advisable that aquarium enthusiasts use personal protective equipment (e.g., goggles, masks, and gloves) whenever working with any type of coral. In cases of ocular exposure, early abundant irrigation and subsequent topical corticosteroid therapy in tandem with topical antibiotic prophylaxis may provide a good outcome. Any patient with corneal infiltrate should be closely monitored, given the risk of possible stromal thinning, melt, and perforation.

\section{Statement of Ethics}

This study protocol was reviewed and the need for approval was waived by Massachusetts Eye \& Ear IRB. Written informed consent to publish this case was obtained from the patient.

\section{Conflict of Interest Statement}

The authors have no conflicts of interest to declare.

\section{Funding Sources}

This manuscript did not receive any funding.

\section{Author Contributions}

T.B., S.O.T., and J.B.C. involved in conceptualization and methodology. T.B. involved in writing - original draft preparation. S.O.T. and J.B.C. involved in writing - reviewing and editing.

\section{Data Availability Statement}

All data generated or analyzed during this study are included herein. Further enquiries can be directed to the corresponding author.

\section{References}

1 Pichon Y. Effects of palytoxin on sodium and potassium permeabilities in unmyelinated axons. Toxicon. 1982; 20(1):41-7.

2 Deeds JR, Schwartz MD. Human risk associated with palytoxin exposure. Toxicon. 2010;56(2):150-62.

3 Sud P, Su MK, Greller HA, Majlesi N, Gupta A. Case series: inhaled coral vapor-toxicity in a tank. J Med Toxicol. 2013;9(3):282-6.

\section{Karger'}


Begaj et al.: Toxic Keratoconjunctivitis from Coral Reef

4 Kodama AM, Hokama Y, Yasumoto T, Fukui M, Manea SJ, Sutherland N. Clinical and laboratory findings implicating palytoxin as cause of ciguatera poisoning due to Decapterus macrosoma (mackerel). Toxicon. 1989; 27(9):1051-3.

5 Patocka J, Nepovimova E, Wu Q, Kuca K. Palytoxin congeners. Arch Toxicol. 2018;92(1):143-56.

6 Vale-Gonzalez C, Pazos MJ, Alfonso A, Vieytes MR, Botana LM. Study of the neuronal effects of ouabain and palytoxin and their binding to Na, K-ATPases using an optical biosensor. Toxicon. 2007;50(4):541-52.

7 Steel DH. Dead man's finger' keratoconjunctivitis. Br J Ophthalmol. 1993;77(1):63.

8 Keamy J, Umlas J, Lee Y. Red coral keratitis. Cornea. 2000;19(6):859-60.

9 Moshirfar M, Khalifa YM, Espandar L, Mifflin MD. Aquarium coral keratoconjunctivitis. Arch Ophthalmol. 2010;128(10):1360-2.

10 Ruiz Y, Fuchs J, Beuschel R, Tschopp M, Goldblum D. Dangerous reef aquaristics: palytoxin of a brown encrusting anemone causes toxic corneal reactions. Toxicon. 2015;106:42-5.

11 Chaudhry NL, Przybek J, Hamilton A, Carley F. Unique case of palytoxin-related keratitis. Clin Exp Ophthalmol. 2016;44(9):853-4.

12 Farooq AV, Gibbons AG, Council MD, Harocopos GJ, Holland S, Judelson J, et al. Corneal toxicity associated with aquarium coral palytoxin. Am J Ophthalmol. 2017;174:119-25.

13 Barbany M, Rossell M, Salvador A. Toxic corneal reaction due to exposure to palytoxin. Arch Soc Esp Oftalmol. 2019;94(4):184-7.

14 Barrett RT, Hastings JP, Ronquillo YC, Hoopes PC, Moshirfar M. Coral keratitis: case report and review of mechanisms of action, clinical management and prognosis of ocular exposure to palytoxin. Clin Ophthalmol. 2021; 15:141-56.

15 Cheung KG, Haynes R, Porter A, O’Hagen S. Palytoxin exposure causing prolonged conjunctivitis and episcleritis without corneal involvement. J Ophthalmol Vis Neurosci. 2017;2(2):15.

16 Wonneberger W, Claesson M, Zetterberg M. Corneal perforation due to palytoxin exposure of domestic zoanthid corals. Acta Ophthalmol. 2020.

17 Gaudchau A, Pfeiffer N, Gericke A. Chemical burns caused by crust anemone. Ophthalmologe. 2019;116(4): 376-9.

18 Jalink MB, van Luijk CM. Corneal melting after moving a tropical aquarium. Ned Tijdschr Geneeskd. 2019;163. 